\title{
3DPredictor: machine learning-based algorithm for prediction of 3D chromatin structure
}

\author{
Belokopytova P. ${ }^{1,2 *}$, Nuriddinov M. ${ }^{1}$, Mozheiko E ${ }^{1}$, Fishman D. ${ }^{2}$, Valiev E. ${ }^{1}$, Fishman V. ${ }^{1,2}$ \\ ${ }^{1}$ Institute of Cytology and Genetics SB RAS, Novosibirsk, Russia \\ ${ }^{2}$ Novosibirsk State University, Novosibirsk, Russia \\ *e-mail: belka2195@mail.ru
}

Key words: Hi-C, machine learning, modelling, promoter-enhancer interactions, chromosomal rearrangements

Motivation and Aim: The question about the exact role of spatial genome organization in regulation of gene expression is still under active debate. However, several studies $[1,2]$ shown that $3 \mathrm{D}$ chromatin folding plays an important role in gene regulation and alterations of spatial chromatin structures can lead to gene misexpression and diseases. It is known that different epigenetic marks and transcriptional factors correlate with various genomic features. In this way modelling helps to reveal main features and patterns underlying 3D genome architecture using epigenetic data as input. Moreover, such approach can be implemented for prediction of consequences of chromosomal rearrangements.

Methods and Algorithms: All data was processed with own scripts written on Python 3.6. Gradient boosting regressor from XGBoost was used as the main machine-learning algorithm

Results: We developed 3DPredictor algorithm which can efficiently predicts 3-dimensional chromatin structures using epigenetic chromatin features as input data. The algorithm could find complex patterns between epigenetic marks and 3D chromatin architecture using machine learning-based approach. Predicted heatmaps are cell-specific and 3DPredictor can be used for prediction of Hi-C maps for different mammal cell types. One of the applications of this method is prediction of chromatin and in particular enhancerpromoter rewiring after chromosomal rearrangements. We used one of well-studied examples of pathological changes caused by 3D chromatin perturbations and implemented our algorithm to predict consequences of such rearrangement. We found that 3DPredictor captures the majority of ectopic interactions. Therefore, this method can be used for prediction of 3D chromatin folding of normal and rearranged genomes.

Conclusion: All code is available at https://github.com/labdevgen/3Dpredictor. 3DPredictor online tool is available at https://genedev.bionet.nsc.ru/Web_3DPredictor/.

Acknowledgements: Supported by the RFBR grant \#18-29-13021. All computations were performed with support of the Computational Cluster of the NSU and Computational Nodes of the Institute of Cytology and Genetics (Budget Project \#0324-2019-0041).

\section{References}

1. Rodríguez-Carballo E. et al. The HoxD cluster is a dynamic and resilient TAD boundary controlling the segregation of antagonistic regulatory landscapes. Genes Dev. Cold Spring Harbor Laboratory Press, 2017. Vol. 31, № 22. P. $2264-2281$.

2. Franke M. et al. Formation of new chromatin domains determines pathogenicity of genomic duplications. Nature. 2016.

Vol. 538, № 7624. P. 265-269. 